\title{
Diacronie
}

Studi di Storia Contemporanea

$\mathrm{N}^{\circ} 29,1 \mid 2017$

"Crash test"

\section{Gianni Fresu, Eugenio Curiel. Il lungo viaggio contro il fascismo}

Andrea Ricciardi

\section{OpenEdition}

Edizione digitale

URL: http://journals.openedition.org/diacronie/5078

DOI: $10.4000 /$ diacronie.5078

ISSN: 2038-0925

Editore

Association culturelle Diacronie

Notizia bibliografica digitale

Andrea Ricciardi, «Gianni Fresu, Eugenio Curiel. Il lungo viaggio contro il fascismo », Diacronie [Online], № 29, 1 | 2017, documento 16, Messo online il 29 mars 2017, consultato il 24 septembre 2020. URL : http://journals.openedition.org/diacronie/5078; DOI : https://doi.org/10.4000/diacronie.5078 


\title{
Diacronie
}

Studi di Storia Contemporanea

29, $1 / 2017$

"Crash test". Continuità, discontinuità, legami e rotture nelle dinamiche della storia contemporanea

\section{RECENSIONE: Gianni FRESU, Eugenio Curiel. Il lungo viaggio contro il fascismo, Roma, Odradek, 2013, 300 pp.}

\author{
A cura di Andrea RICCIARDI
}

Per citare questo articolo:

RICCIARDI, Andrea, «RECENSIONE: Gianni FRESU, Eugenio Curiel. Il lungo viaggio contro il fascismo, Roma, Odradek, 2013, 300 pp.», Diacronie. Studi di Storia Contemporanea : "Crash test". Continuità, discontinuità, legami e rotture nelle dinamiche della storia contemporanea, 29, 1/2017, 29/3/2017,

URL: < http://www.studistorici.com/2017/3/29/ricciardi_numero_29/ >

Diacronie Studi di Storia Contemporanea $\rightarrow$ http://www.diacronie.it

Rivista storica online. Uscita trimestrale.

redazione.diacronie@hotmail.it

Comitato di direzione: Naor Ben-Yehoyada - João Fábio Bertonha - Christopher Denis-Delacour - Maximiliano Fuentes Codera Anders Granås Kjøstvedt - John Paul Newman - Deborah Paci - Niccolò Pianciola - Spyridon Ploumidis - Wilko Graf Von Hardenberg

Comitato di redazione: Jacopo Bassi - Luca Bufarale - Gianluca Canè - Fausto Pietrancosta - Alessandro Salvador - Matteo Tomasoni Diritti: gli articoli di Diacronie. Studi di Storia Contemporanea sono pubblicati sotto licenza Creative Commons 3.0. Possono essere riprodotti e modificati a patto di indicare eventuali modifiche dei contenuti, di riconoscere la paternità dell'opera e di condividerla allo stesso modo. La citazione di estratti è comunque sempre autorizzata, nei limiti previsti dalla legge. 


\title{
16/ RECENSIONE: Gianni FRESU, Eugenio Curiel. Il lungo viaggio contro il fascismo, Roma, Odradek, 2013, 300 pp.
}

\author{
A cura di Andrea RICCIARDI
}

Eugenio Curiel è una delle figure più interessanti dell'antifascismo italiano, avvolta in un'atmosfera di drammatica incompiutezza. Venne infatti assassinato dai fascisti a Milano il 24 febbraio 1945, poco più di due mesi prima della Liberazione, a soli 33 anni (non ancora compiuti). Riconosciuto da un delatore, l'ex confinato di Ventotene Amilcare Rolando, mentre si recava ad incontrare la sorella Grazia, tentò una disperata fuga che si concluse con il suo ferimento. Aveva detto in più circostanze che era necessario non farsi catturare vivi, evitando così le orribili torture di "Villa triste" e della famigerata banda Koch, che avrebbero potuto portare chiunque a rivelare informazioni sull'identità e i movimenti dei compagni e a compromettere così la struttura clandestina del PCI, in cui militava con grande passione e spirito di abnegazione. Fece dunque appello alle sue ultime forze per rialzarsi e, di fatto, per farsi sparare una seconda e definitiva volta.

Alla fine di un volume molto corposo, ricco di sollecitazioni ma di non facile lettura, diviso in tre capitoli e corredato da due testimonianze di Gianni Cervetti e Aldo Tortorella (riportate integralmente in appendice, ma parzialmente riproposte in varie parti del testo), Fresu scrive che Curiel

morì alla vigilia della vittoria finale, nel gelido febbraio milanese, senza poter vedere i colori di una primavera a lungo attesa e per la quale tanto aveva lottato, la liberazione. Vide e contribù̀ ad accelerare il tramonto della dittatura, non poté assaporare l'alba di una nuova democrazia, forse anche in questo particolare sta il fascino tragico del suo destino, drammaticamente segnato dalla violenza del fascismo'.

Non furono pochi gli antifascisti di diverso colore che persero la vita in vista del successo finale, qualcuno tentando di sottrarsi alla morte pianificata dai nazisti nei campi di sterminio (come il socialista Ermanno Bartellini, primo "maestro" di politica di Leo Valiani, e l'azionista Luigi

${ }^{1}$ Cfr. FRESU, Gianni, Eugenio Curiel. Il lungo viaggio contro il fascismo, Milano, Odradek, 2013, p. 241. 
Cosattini), altri perché assassinati tra il 1944 e i primi mesi del 1945 in circostanze talvolta strazianti. Tra questi, solo per portare qualche esempio, si possono ricordare Duccio Galimberti, Eugenio Colorni (amico di Curiel), Bruno Buozzi e Leone Ginzburg.

Curiel morì da comunista, a stretto contatto con Giorgio Amendola, Gillo Pontecorvo e altri compagni più giovani che, durante la fase più difficile e cruenta della Resistenza, ne riconobbero le qualità di leader e la profondità di pensiero. Tuttavia, nel corso della sua esistenza, egli attraversò momenti diversi nei quali la sua naturale tendenza all'approfondimento e alla ricerca lo mise in contatto con correnti di pensiero diverse dal marxismo-leninismo e lo portò a lavorare al fianco di importanti esponenti dell'antifascismo socialista (quello con Giustizia e Libertà si rivelò, invece, un incontro mancato).

Eugenio Curiel, nato da Giulio e Lucia Limentani, genitori ebrei esponenti della dinamica borghesia triestina, secondo Fresu si deve considerare interprete di una generazione (titolo del primo capitolo), quella cioè di chi crebbe sotto il fascismo, colse precocemente il grande inganno che si celava dietro un'organicità di sistema e di idee solo apparente e si mise pienamente in gioco per costruire un futuro diverso, all'insegna della coscienza e della libertà. Già dal titolo del libro, si capisce che Curiel è avvicinato per vari aspetti a Ruggero Zangrandi, di tre anni più giovane, autore del celebre volume autobiografico Il lungo viaggio attraverso il fascismo ${ }^{2}$. Il libro viene evocato indirettamente da Fresu: pubblicato nel 1962, ha rappresentato (e rappresenta ancora oggi) un documento di quel travaglio generazionale che coinvolse molti ragazzi, inevitabilmente immersi nel regime di Mussolini ma, nel corso degli anni Trenta, sempre più orientati a metterlo in discussione evidenziando le stridenti contraddizioni tra i roboanti annunci inneggianti alla rivoluzione fascista e la realtà di un paese conformista. Un paese in cui la gestione del potere era rimasta nelle mani dei grandi capitalisti e degli agrari, che avevano finanziato lo squadrismo in funzione antisocialista e che avevano, poi, tratto tutti i possibili vantaggi dall'azzeramento della dialettica sociale e dallo sfruttamento dei lavoratori.

Proprio il primo capitolo appare particolarmente stimolante per il modo in cui Fresu si sofferma sul giovane Curiel alla ricerca di un'identità, culturale prima che politico-ideologica. Molto dotato negli studi, con una predilezione per le discipline scientifiche e una naturale tendenza alla cultura enciclopedica, Eugenio si interessò anche di pittura, letteratura, teatro e musica, come sottolineato nella biografia di Briamonte ${ }^{3}$ (che, pur essendo un lavoro pubblicato da quasi quarant'anni, rimane molto importante e viene spesso utilizzato da Fresu come fonte).

Curiel era dotato di una personalità forte grazie alla quale non subì grandi condizionamenti dalla famiglia ma, nel contempo, fu sempre molto legato a tutti i suoi componenti. Fece quasi da secondo

\footnotetext{
${ }^{2}$ Cfr. ZANGRANDI, Ruggero, Il lungo viaggio attraverso il fascismo. Contributo alla storia di una generazione, Milano, Feltrinelli, 1962.

${ }^{3}$ Cfr. BRIAMONTE, Nando, La vita e il pensiero di Eugenio Curiel, Milano, Feltrinelli, 1979.
} 
padre alle due sorelle Grazia e Gigliola e al fratello Sergio, costruì un rapporto intenso anche con la nipotina Luciana (da lui chiamata Puck), come testimoniato dalla sua compagna Bianca Diodati. Per Fresu, «nonostante la solidità di questi rapporti, Eugenio non ebbe remore ad andare contro le indicazioni paterne per assecondare le proprie convinzioni politico-sociali» ${ }^{4}$. Una cugina più giovane, Matilde Finzi Bassani, fu un'altra parente per lui importante, che confessò a Eugenio la precoce militanza antifascista, guadagnandosi così la sua considerazione. Curiel la presentò quindi a Colorni e a Guido Goldschmied, suoi compagni di lotta con cui Matilde intraprese fecondi rapporti di collaborazione.

Terminati gli studi al liceo scientifico nel 1929, con un anno di anticipo, Eugenio si iscrisse al biennio di ingegneria, ospitato a Firenze dallo zio Ludovico Limentani, filosofo, che contribuì ad alimentare l'interesse del giovane per quella disciplina. Iscrittosi al politecnico di Milano nel 1931, nonostante il suo approccio "problematico" allo studio, Curiel mostrò una propensione particolare per le scienze fisico-matematiche e per la ricerca scientifica "pura". Ma la crisi economica, conseguenza indiretta del crollo della borsa di New York del 1929, condizionò il corso degli studi di Eugenio, visto che la famiglia subì un tracollo - comune a una parte rilevante dei ceti medi - che, per usare le parole dell'autore, la condusse anche «al declassamento dello status sociale». Ciò ebbe una conseguenza rilevante sulla crescita di Curiel, «imprimendo una sua maturazione accelerata e un'assunzione di responsabilità e doveri verso la famiglia in difficoltà» ${ }^{5}$.

Curiel decise così di abbandonare Milano e di tornare dallo zio a Firenze, dove si iscrisse al corso di laurea in Fisica. Scrive Fresu:

Ma non erano solo i problemi economici ad agitare il giovane Curiel, maturava infatti in lui un turbamento nervoso e morale che se per un verso rafforzò la sua tendenza all'introspezione, dall'altro lo spinse a cercare nuovi orizzonti, sia culturali che esistenziali6.

Da qui l'incontro con le discipline antroposofiche di Rudolf Steiner, che si tradusse in una crescita intellettuale autonoma dall'humus culturale e religioso della famiglia, pur rivelandosi un percorso filosofico e spirituale non lineare e, per certi aspetti, sofferto. Un altro incontro determinante per il corso dell'esistenza di Curiel fu quello con il fisico Bruno Rossi (con cui Eugenio stava preparando la tesi di laurea nel 1933), assistente a Firenze quando ottenne la cattedra a Padova, dove fu seguito dal giovane laureando. Nel luglio dello stesso 1933, dopo aver provvisoriamente abbandonato gli studi per insegnare nelle scuole elementari, Curiel si laureò proprio grazie alla pressioni di Rossi e dei familiari, in questo caso ascoltati da Eugenio. Il 110 e lode,

\footnotetext{
${ }^{4}$ Cfr. FRESU, Gianni, Eugenio Curiel. Il lungo viaggio contro il fascismo, cit., p. 20.

${ }^{5}$ Ibidem, p. 22.

${ }^{6}$ Ibidem.
} 
in una fase di adesione "totale" allo steinerismo, non placò il suo tormento interiore ma sopravvenne in un periodo nel quale, proprio grazie ai fertili dialoghi interni al gruppo steineriano, Curiel definì con maggiore organicità il suo antifascismo. É a Padova che Eugenio incontrò Atto Braun, amico d'infanzia a Trieste, e Renato Mieli, un giovane ebreo nativo di Alessandria d'Egitto. Con loro, e con Guido Goldschmied, Curiel avrebbe formato la cellula comunista nella città veneta.

Un aspetto centrale della strategia di Curiel fu l'idea che il fascismo potesse e dovesse essere combattuto innanzitutto "dall'interno", cioè utilizzando canali e strumenti legali per costruire un rapporto solido con i giovani. Secondo la testimonianza di Opocher ${ }^{7}$, divenuto poi azionista, nonostante la diffidenza di vari antifascisti, Curiel aveva compreso che era necessario erodere la base di consenso del regime senza aver timore di confrontarsi con la dissidenza fascista e, quindi, di agire all'interno di un terreno teoricamente ostile. Da qui l'impegno profuso nella rivista «Il Bò» e l'esperienza nei GUF, a cui seguì quella nel Fronte della Gioventù. Un'organizzazione, questa, ideata da Pajetta in accordo con Longo e che vide coinvolti, tra gli altri, Pontecorvo, Vittorini, Tortorella e Raffaele De Grada. Curiel, nel completare la sua formazione, entrò in contatto con GL, le cui posizioni presentavano qualche affinità con il suo approccio alla lotta. Ma l'incontro con Mario Paggi a Milano lo deluse, portandolo ad intensificare i rapporti con l'amico Braun e ad approfondire i classici del marxismo-leninismo. Una tendenza che avrebbe caratterizzato Curiel anche durante il confino $^{8}$ quando, oltre a Marx ed Engels, lesse e discusse Antonio Labriola, Lukács, Antonio Banfi e Bucharin, confrontandosi con antifascisti di diversa estrazione ideologico-culturale.

Entrato in contatto con la direzione del PCd'I nel 1936, Curiel si recò a Parigi nel marzo del 1937. Qui iniziò a costruire un rapporto (non proprio lineare) con il centro estero e, per alcune settimane, frequentò Emilio Sereni, Donini e Grieco. Con loro discusse la situazione italiana e, rientrato a Padova, «orientò i compagni a un lavoro legale di massa attraverso la penetrazione nelle stesse realtà sociali del regime tra lo sconcerto della cellula comunista composta da giovani che fremevano

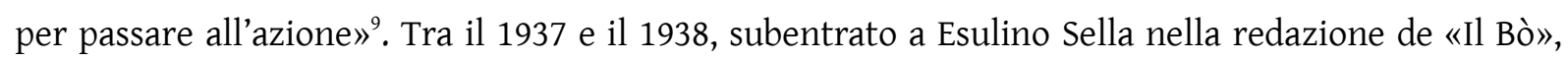
scrisse per la rivista 54 articoli trattando di questioni sindacali e, più in generale, di problemi del lavoro. Oltre a questo canale, Curiel si propose di intercettare i malumori dei giovani intellettuali partecipando ai Littoriali nello stesso 1937 (a Napoli) e nel 1938 (a Palermo).

Fresu vede in questa strategia una consonanza con la linea intrapresa dal centro estero del PCd'I.

\footnotetext{
${ }^{7}$ Ibidem, p. 32.

${ }^{8}$ Curiel fu arrestato a Trieste il 24 giugno 1939, tradotto in carcere a Milano e condannato il 13 gennaio 1940 a cinque anni di confino. Fu quindi trasferito a Ventotene, che lasciò il 21 agosto 1943. Cfr. la voce su Eugenio Curiel redatta da Nicola Briamonte per il Dizionario Biografico degli Italiani (vol. 31, 1985), reperibile su internet, URL: < http://www.treccani.it/enciclopedia/eugenio-curiel_(Dizionario-Biografico)/ > [consultato il 16 febbraio 2017].

${ }^{9}$ Cfr. FRESU, Gianni, Eugenio Curiel. Il lungo viaggio contro il fascismo, cit., p. 39.
} 
Nei fatti, Curiel seppe raccogliere l'intuizione sviluppata da Ruggero Grieco tra il 1934 e il '36, ossia, puntare proprio sulle nuove generazioni e sugli operai per mettere in crisi il fascismo ${ }^{10}$.

Un altro aspetto centrale del modo di concepire la lotta antifascista da parte di Curiel fu la sua tendenza a promuovere uno stretto rapporto con i socialisti del centro interno, cosa che gli attirò critiche e persino sospetti ma che, come spiega Fresu, era coerente con la strategia dei fronti popolari promossa dal VII Congresso del Comintern. La consonanza di vedute con i socialisti fu tale che Merli sostenne che Curiel aderì realmente al PCd'I solo durante il confino, mentre nella fase precedente fu molto più in sintonia con Basso, Colorni e Morandi ${ }^{11}$. Dunque il modo di declinare il rapporto tra i due partiti operai, da tema politico, sarebbe divenuta poi una controversa questione storiografica visto che, per Spriano, Curiel scelse invece di aderire al PCd'I addirittura nel $1935^{12}$.

In questa sede non è possibile tracciare un quadro di tutte le considerazioni espresse dall'autore su questo doppio binario (politico e storiografico), né dar conto con organicità delle numerose testimonianze inserite nel libro che, a tratti, considerati anche i continui salti temporali, appare un po' disordinato. Questa sensazione emerge inoltre perché l'autore, forse per contestualizzare al meglio le riflessioni e l'attività di Curiel, si sofferma a fondo sulla storia complessiva del PCd'I, talvolta accantonando il percorso individuale dello stesso Curiel. La tendenza appare manifesta nel secondo capitolo, intitolato significativamente Svolte e contro svolte: $i$ comunisti italiani di fronte al fascismo. Curiel è sostanzialmente assente dalla narrazione, talvolta compare solo per verificare se la sua linea fosse più o meno coerente con le direttive dei vertici e, in particolare, di quel centro estero messo in discussione da Mosca nel 1937, quando Berti fu inviato a Parigi.

L'equilibrio tra la vicenda individuale e la più ampia storia del partito è maggiore nel terzo capitolo che, intitolato Alla testa di una generazione, si apre con una tripartizione cronologica dei nodi essenziali del contributo di Curiel alla storia dell'antifascismo, elaborata da Eugenio Garin:

1) l'utilizzo degli spazi legalitari ancora rimasti per la lotta contro il fascismo, operando tra i giovani inquadrati nelle organizzazioni universitarie e gli operai nei sindacati fascisti (1937-39); 2) la ricerca di un patrimonio teorico peculiare, attraverso lo studio del marxismo e della storia d'Italia in rapporto al suo processo di unificazione, negli anni del confino a Ventotene (1940-43); 3) la mobilitazione politica e militare dei giovani nella lotta di liberazione nazionale, all'interno della prospettiva socialista e di rinnovamento democratico del Paese $(1943-45)^{13}$.

\footnotetext{
${ }^{10}$ Ibidem, p. 43.

${ }^{11}$ Ibidem, p. 61.

${ }^{12}$ Ibidem, p. 65.

${ }^{13}$ Ibidem, p. 159. Il volume di Garin da cui è tratta la citazione è GARIN, Eugenio, Intellettuali italiani del XX secolo, Roma, Editori Riuniti, 1974.
} 
Proprio a quest'ultima stagione della vita di Curiel, Fresu dedica attenzione evidenziando, da una parte, gli intensi rapporti politici e personali sviluppati con vari compagni (tra cui l'amico ritrovato Colorni, Colombi, Di Vittorio, Pietro Grifone, Giovanni Pesce, Secchia, Scoccimarro, Longo) e, dall'altra, la natura delle sue riflessioni che, sempre secondo l'autore, presentarono rilevanti punti di contatto, sia pure inconsapevoli, con Gramsci e con il Togliatti della svolta di Salerno e del partito nuovo. 


\section{L'AUTORE}

Andrea RICCIARDI (collabora dal 2000 con l'Università degli Studi di Milano, dove si è laureato, ha conseguito il dottorato di ricerca in Storia della società e delle istituzioni nell'Europa contemporanea, ha lavorato per quattro anni come assegnista e, attualmente, è docente a contratto di Storia contemporanea. Dal 2010 è membro della redazione de «Il Mestiere di Storico» e dal 2011 del comitato di direzione degli «Annali della Fondazione Ugo La Malfa». Si occupa di storia politica e culturale, con particolare riferimento a figure e vicende del movimento operaio e dell'antifascismo. Oltre a vari saggi pubblicati in riviste di settore e volumi collettanei, ha scritto e curato libri su Leo Valiani, Gino Giugni, Vittorio Foa, Riccardo Lombardi, Antonio Giolitti e Luigi Longo. Nel 2013 ha ottenuto l'ASN per professore associato di Storia contemporanea (11/A3).

URL: < http://www.studistorici.com/progett/autori/\#Ricciardi > 